Edvard Govekar, Alexander Kuznetsov, Anže Jerič, Drop on demand generation from a metal wire by means of an annular laser beam, Journal of Materials Processing Technology,

Volume 227,

2016,

Pages 59-70,

ISSN 0924-0136,

(C) 2015. This manuscript version is made available under the CC-BY-NC-ND 4.0 license http://creativecommons.org/licenses/by-nc-nd/4.0/

Link to published article:

http://www.sciencedirect.com/science/article/pii/S0924013615300777

DOI: https://doi.org/10.1016/j.jmatprotec.2015.07.026 


\title{
Drop on demand generation from a metal wire by means of an annular laser beam
}

Edvard Govekar ${ }^{a^{*}}$, Alexander Kuznetsov ${ }^{a}$, Anže Jerič ${ }^{a}$

${ }^{a}$ University of Ljubljana, Faculty of Mechanical Engineering, Aškerčeva cesta 6, SI-1000 Ljubljana, Slovenia

* Corresponding author. Tel.: +386 14771 606; fax: +386 14771 651. E-mail address: edvard.govekar@ fs.uni-lj.si.

\begin{abstract}
In the paper a novel system for drop-on-demand (DoD) generation from a metal wire is presented, whose main component is a newly developed laser droplet generation head, consisting of annular laser beam shaping optics and a wire feeding system. In the pendant droplet formation phase of the DoD generation, a laser pulse is used to melt the wire-end, which is fed into the focus of an annular laser beam. The formed pendant droplet is then detached by means of a detachment pulse, which induces Rayleigh - Plateau instability of the molten column of wire above the neck of the pendant droplet. The main process parameters, including the laser pulse and wire feeding parameters as well as the additional parameters which influence particular phases of the DoD generation process, have been identified. The empirical correlations between the influencing process parameters and the droplet characteristics, including droplet diameter and temperature, were determined, based on the analysis of high speed IR records of the process, images being acquired by an optical microscope and temperature data being acquired by pyrometers. As an example, DoD generation from a commercially pure $99.6 \% \mathrm{Ni}$ wire (Nickel 200) of $0.6 \mathrm{~mm}$ diameter is considered. It is shown that droplets with diameters ranging from 0.85 to $1.25 \mathrm{~mm}$ can be generated, with a resolution of $50 \mu \mathrm{m}$ and a standard deviation of $15 \mu \mathrm{m}$. The temperature of the detached droplet remains above the melting point of the $\mathrm{Ni}$ wire, and increases with the droplet diameter within the range from $1650{ }^{\circ} \mathrm{C}$ to $1750{ }^{\circ} \mathrm{C}$. Some examples of Ni droplets deposited on a Ti sheet surface are presented, with the aim of demonstrating the capability of the proposed system, and motivating further applications in which drops on demand having a high temperature and a precisely defined diameter need to be generated, while limiting the thermal loading of the surroundings.
\end{abstract}

Keywords: Droplet generation; Drop-on-demand; Metal wire; Annular laser beam

\section{Introduction}

Metal droplets are the basic elements which are involved in the droplet-based innovative manufacturing technologies (Chun et al. 1993, Liu, 2000) which are in high demand in many different industrial applications. A 
particularly challenging droplet-based manufacturing technology that is still under development is printing technology (Liu and Orme, 2001), which can be used for industrial applications such as rapid prototyping (Zhang et al., 2003), 3D structuring (Yamaguchi et al., 2000), and the freeform fabrication of components (Qi et al., 2012). Metal droplets can also be used for narrowly specialized micro-casting applications (Zarzalejo et al. 1999). Additionally, high energy droplets can be applied for the high-temperature-resistant lead-free joining of electronic contacts (Dreizin, 1997), for the joining of dissimilar materials (Albert et al., 2011) and of coated, temperature-sensitive materials (Jerič et al.,2009) In order to fulfil the requirements of particular application, various droplet generation systems and processes, have been developed.

For the generation of low melting point droplets $\left(T_{\text {melt }}<427^{\circ} \mathrm{C}\right)$, materials in the liquid phase are used the most frequently. Methods of continuous droplet generation are based on liquid metal stream break up due to induced stream instability which can be caused by low-energy mechanical vibration (Liu and Omre 2001), or by electromagnetic forces (Shimasaki and Taniguchi, 2009). In DoD generation a liquid metal is squeezed out through a nozzle by applying a high energy pulse which can be generated by a piezoelectric actuator (Wehl et al., 2003), a vibration plunger (Sohn and Yang, 2005), or a pneumatic actuator (Cheng et al., 2005).

However, in the case of high-temperature melting point droplets $\left(T_{\text {melt }}>427^{\circ} \mathrm{C}\right)$, materials that are initially in the solid phase are more suitable. In the most commonly used method in the case of continuous droplet generation based metal transfer, which is used in gas metal arc welding (GMAW), an electric arc is applied between the fed wire electrode and the droplet deposition or joining spot (Hu and Tsai, 2007). DoD generation can in this case be achieved by controlling the arc (Wu et al., 2004) or by wire feeding control (Zhang et al., 2002). A TIG arc based micro welding system has been employed for 3D structuring (Horii et al., 2009), where the arc discharge current is used to control the properties of deposited micro beads (Terakubo et al., 2005). The main drawbacks of these methods are the large heat affected area at the joining spot and the high thermal loadings in the surroundings due to the electrical arc. As such, arc-based methods are not suitable for droplet based applications in which temperature sensitive components are used. The electrical arc between the wire and the joining spot can be omitted by using cathodes around the fed consumable wire anode (Conway, 2002). However, the system has limits in the providing of sufficiently small droplets and the presence of high thermal loadings in the surroundings, which still prevents applications in the electronics industry where temperaturesensitive components are used. 
Considering the drop-on-demand (DoD) generation process, which fulfils the requirements for the application of high melting point materials and low thermal loading in the surroundings, various laser based droplet generation methods have been proposed. The application of a laser beam besides the processing of high melting point materials has several other benefits, including precise control of the temporal and spatial energy input, and a short time of heating impact, which results in a small heat-affected area and low thermal loading of the surroundings. The proposed laser based methods differ with regard to the form of the source material being used for droplet generation, and include preformed metal balls (Quentin et al., 2013), metal foil (Jeromen et al., 2014), and metal wire (Jahrsdörfer et al., 2003). Using source material in the form of a wire provides material and flexibility and can be used to generate droplets with different diameters (Govekar et al., 2007). Laser droplet generation from a wire has good proven potential for various droplet-based spot and continuous micro-joining applications (Govekar et al,. 2009), especially for the joining of dissimilar materials, and for thin foils and temperature sensitive metallizations in the electronics industry (Albert et al. 2011), as well as for the joining of zinc-coated metal sheets (Jerič et al., 2009).

In the conventional LDG process a Gaussian laser beam pulse is used to melt the end of the fed metal wire, and to detach the formed pendant droplet from the solid part of the wire-end. In past research into the use of LDG process using a metal wire, several LDG prototype systems using a single beam (Jerič et al., 2009) and several (three) laser beams (Jahrsdörfer et al., 2003), have been developed. The main disadvantages of these systems are the uneven distribution of the laser beam light at the wire-end circumference in the case of a single beam, and the complexity of the system in the case of three laser beams, what can lead to high instability of the LDG process (Krese et al., 2011). Using a Gaussian laser beam, the forced detachment of a pendant droplet can be achieved on demand by the force of the vapour recoil pressure (Kokalj et al., 2006), but this method is accompanied by droplet splashes, high variance of the droplet diameter, and a lateral scatter of the detached droplets.

To avoid the above-mentioned problems, a novel annular laser beam droplet generation (A-LDG) system has been designed. The system uses a single annular (i.e. ring-shaped) laser beam that is guided coaxially with the wire and focused onto the circumference of the wire-end. The annular shape of the laser beam ensures uniform heating of the wire-end around its whole circumference. Additionally, due to the properties of the annular laser beam, forced droplet detachment on demand is achieved by the Rayleigh-Plateau (R-P) instability of the molten wire column above the pendant droplet neck (Kuznetsov et al., 2014). This results in a significant reduction in the extent of droplet splashes, lower droplet diameter variance, and lower lateral scatter of the detached droplets. 
In addition, the use of an annular laser beam, coaxially with the wire, enables pre- and post-heating of the droplet deposition spot.

In this paper the novel A-LDG system and process are presented. With this aim, the experimental setup and the system for A-LDG from a metal wire are first described. Next, the DoD generation process is analysed in detail with respect to the process phases and parameters. The results of A-LDG process characterization and of the correlations between the process parameters and the droplet characteristics are then presented for the case of DoD generation from a commercially pure $99.6 \% \mathrm{Ni}$ wire (Nickel 200) having a diameter of $0.6 \mathrm{~mm}$ was used. Preceding the conclusions, examples of Ni droplets deposited on a Ti sheet surface are shown in order to demonstrate the capability of the proposed A-LDG system, and potential applications of DoD generated droplets.

\section{Experimental setup}

The experimental setup for A-LDG from a metal wire is shown schematically in Fig. 1(a). The setup consists of a laser source system, beam shaping optics, a wire feeding system, and the process monitoring and control system, which are here below described in greater detail.
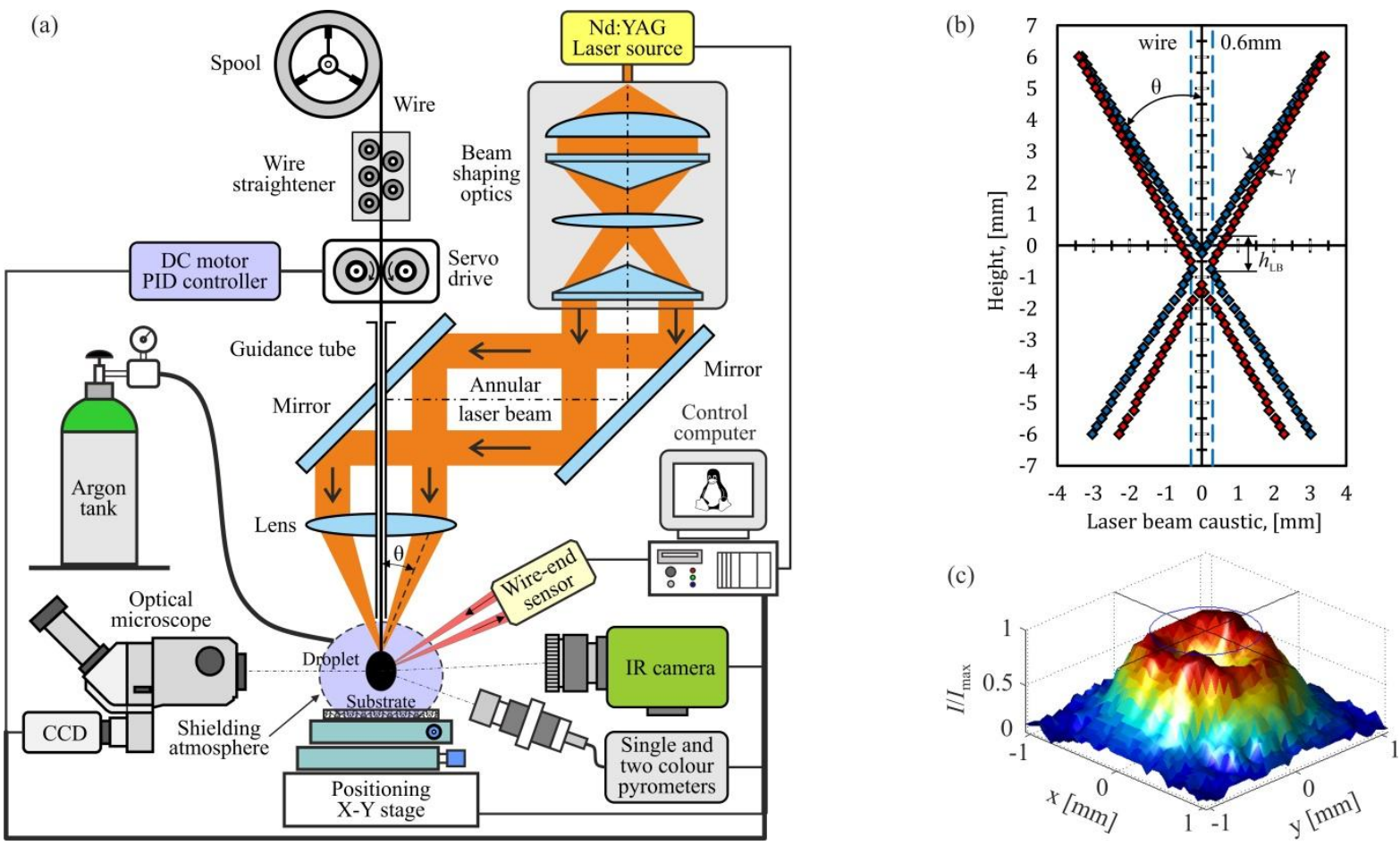

Fig. 1. (a) Scheme of the annular LDG system; (b) Caustic of the annular laser beam; (c) Example of the annular laser beam intensity distribution. 


\subsection{The laser source system and beam shaping optics}

The laser source used in the experimental setup is an industrial Nd:YAG pulsed laser of $\lambda=1064 \mathrm{~nm}$ wavelength, with Gaussian distribution of the laser beam intensity. The laser emits pulses with time-dependent profile of power in the range from 0.5 to $8 \mathrm{~kW}$ and a duration from 0.3 to $20 \mathrm{~ms}$. The maximum pulse frequency is $300 \mathrm{~Hz}$ with an average power of $250 \mathrm{~W}$. The energy limit of a single laser pulse is $30 \mathrm{~J}$.

The emitted laser beam is guided through the beam shaping optics, where it is collimated and transformed into the annular shape by the pair of axicons and a convergent lens. The annular beam is guided coaxially to the axis of the wire by means of two reflective mirrors, and finally focused on the wire-end circumference by a focusing lens.

The measured annular laser beam caustic is presented in Fig. 1(b). The annular laser beam can be described as a hollow cone with a thin wedge-shaped wall. The wedge angle of the wall is $\gamma=2.5^{\circ}$. The vertex half-angle of the cone, i.e. the half-angle of the beam convergence is $\Theta=27^{\circ}$. The height $h_{\mathrm{LB}}$ of the annular laser beam spot projected vertically onto the wire surface depends on the wire diameter $D_{\mathrm{w}}$. In the case of $D_{\mathrm{w}}=0.6 \mathrm{~mm}$ the height $h_{\mathrm{LB}}$ amounts to $0.80 \mathrm{~mm}$. The corresponding annular laser beam relative intensity distribution $I / I_{\max }$ captured by the IR camera slightly above the focus position is shown in Fig. 1(c).

\subsection{The wire feeding system}

In order to generate a droplet, the wire-end has to be fed coaxially into the annular laser beam focus. For this purpose a wire feeding system consisting of a spool, a wire straightener, a wire feeding servo drive, and a guidance tube, is used. For precise feeding of the wire during the LDG process, the wire feeding servo drive is closed loop controlled. The servo drive provides a resolution of the wire displacement of $20 \mu \mathrm{m}$, with a maximum wire feeding acceleration / deceleration of $30 \mathrm{~m} / \mathrm{s}^{2}$, and a peak velocity of $0.35 \mathrm{~m} / \mathrm{s}$. Wires with diameters within the range from 0.25 to $1 \mathrm{~mm}$ can be used.

Coaxiality of the wire and the laser beam is achieved by means of a guidance tube, which is used to guide the wire into the annular laser beam focus. To ensure sufficiently precise lateral positioning of the wire-end, the wire that is unwound from the spool is straightened by a 4-plane wire straightener. 


\subsection{The process monitoring and control system}

A-LDG is a complex process which requires in-process monitoring and control with the aim of generating droplets having the desired properties. In order to monitor and control the A-LDG process, the experimental setup (Fig. 1a) was equipped with a high speed IR camera, a CCD camera with an optical microscope, two pyrometers, and a laser reflection sensor.

A high speed IR camera with a wavelength range of between 3.5 and $5 \mu \mathrm{m}$ was used to characterize the A-LDG process dynamics and to measure the pendant droplet diameter $D_{\text {pdr }}$ and the detached droplet diameter $D_{\mathrm{dr}}$ based on acquired IR image sequences of the process. The used frame resolution was $64 \times 32$ pixels, and the frame rate was 3600 frames per second. Additionally, the pendant droplet diameter $D_{\text {pdr }}$, and the diameter $D_{\text {rp }}$ of the wireend remaining part, i.e. of the small droplet which stays attached to the wire-end after pendant droplet detachment, were extracted from the images acquired by the CCD camera with image resolution 576x720 pixels. To assure high measurement accuracy of $D_{\mathrm{pdr}}$ and $D_{\mathrm{rp}}$ the CCD camera was attached to an optical microscope which provided a magnification of 125 times. With the aim of increasing the measurement accuracy of $45.5 \mu \mathrm{m}$ provided by the IR camera in the case of the detached droplet diameter $D_{\mathrm{dr}}$, a method based on image segmentation and the use of a correction factor provided from the measurements of the pendant droplet diameter $D_{\text {pdr }}$ with the IR and CCD cameras was used.

During the A-LDG process the surface temperature $T_{\mathrm{ws}}(t)$ of the wire-end was measured at the spot of the annular laser beam focus, by means of a two-colour pyrometer of spectral wavelengths $\lambda_{1}=1.65$ and $\lambda_{2}=2 \mu \mathrm{m}$. The pyrometer had a temperature measurement range from 500 to $3000{ }^{\circ} \mathrm{C}$, a time response of $0.2 \mathrm{~ms}$, and a minimum measuring spot diameter of $0.4 \mathrm{~mm}$. Based on $T_{\mathrm{ws}}(t)$, information about the initial surface temperature $T_{\text {гр }}$ of the wire-end remaining part, and about the surface temperature $T_{\mathrm{pdr}}$ of the formed pendant droplet, were acquired. The surface temperature $T_{\mathrm{dr}}$ of the detached droplet was measured by a single-colour pyrometer with a spectral wavelength of $\lambda_{3}=2.2 \mu \mathrm{m}$ and a temperature range from 300 to $2300{ }^{\circ} \mathrm{C}$. The corresponding pyrometer time response and the minimum measuring spot diameter were $0.18 \mathrm{~ms}$ and $0.8 \mathrm{~mm}$, respectively. The information obtained from the laser reflection sensor was used, together with the control computer and the wirefeeding system, to ensure the proper wire-end vertical position $L_{\mathrm{we}}$, with a resolution of $25 \mu \mathrm{m}$. Additionally, from the CCD image the distance $L_{\mathrm{dn}}$ between the pendant droplet neck and the annular laser beam focus can be monitored either in- or post-process. 
All the mentioned monitoring data (IR image records, $\left.D_{\mathrm{pdr}}, D_{\mathrm{rp}}, D_{\mathrm{dr}}, T_{\mathrm{ws}}(t), T_{\mathrm{pdr}}, T_{\mathrm{rp}}, T_{\mathrm{dr}}, L_{\mathrm{we}}, L_{\mathrm{dn}}\right)$ were acquired by a data acquisition system which was embedded in the control computer. The data acquisition system was also used to acquire the emitted power $P(t)$ of the laser pulse with a resolution of $40 \mathrm{~W}$. Based on the pre-defined desired droplet characteristics $\left(D_{\mathrm{dr}}, T_{\mathrm{dr}}\right)$ and the selected acquired data $\left(D_{\mathrm{rp}}, T_{\mathrm{rp}}\right)$ the control computer is able to define proper values of the A-LDG process parameters $\left(P(t), V_{\mathrm{w}}(\mathrm{t}), L_{\mathrm{we}}, L_{\mathrm{dn}}\right)$ and to provide the corresponding control and contemporized triggering signals for synchronization of the laser source, wire feeding, and the $X-Y$ positioning stage.

\subsection{The laser droplet generation head}

The described laser beam shaping optics, the reflective mirrors with focusing lens, the wire feeding system consisting of a wire spool, a straightener, and a wire feeding servo drive, the wire guidance tube, the two pyrometers, the wire-end laser reflection sensor and the microscope with CCD camera are assembled together, by means of an aluminum housing, into a compact autonomous laser droplet generation head, as shown in Fig. 2. In order to prevent metal oxidation during the A-LDG process, the head is equipped with a coaxial nozzle in order to supply the argon shielding gas around the wire-end and the droplet deposition surface.

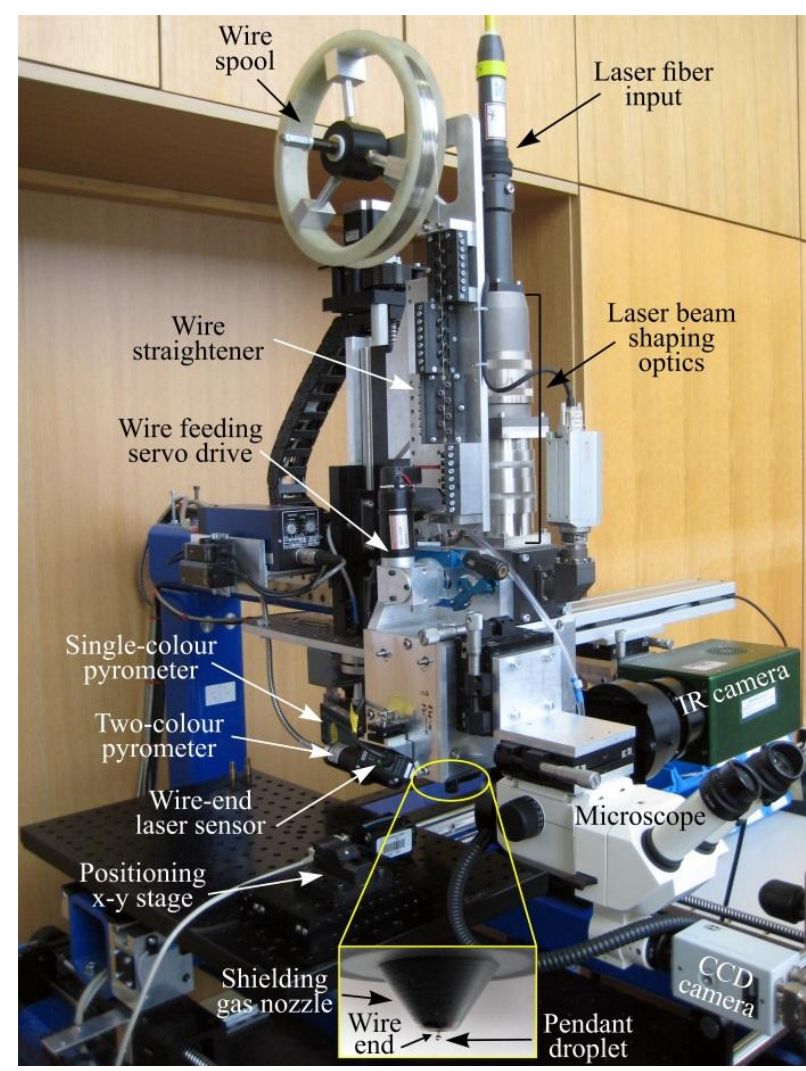


Fig. 2. Photo of the annular laser droplet generation head.

\section{The laser drop-on-demand generation process}

The goal of the laser drop on demand generation (LDoDG) process is to generate a droplet with predefined characteristics (i.e. a droplet with a diameter Ddr and a temperature Tdr) at the moment of demand. In the following, the process of LDoDG from a metal wire is described in detail, and the process parameters are identified.

Phenomenologically viewed, the LDoDG process consists of two phases: the pendant droplet formation phase, and the droplet detachment phase. The phases are further divided into a set of sequential stages with corresponding stage outcomes as is shown schematically in Fig. 3(a-e). The main LDoDG process parameters are the laser pulse power profile $P(t)$ and the wire feeding velocity profile $V_{\mathrm{w}}(t)$. In Fig. 3(f) the laser pulse power profile $P(t)$ and the wire feeding velocity are shown schematically with respect to the defined process stages. A comprehensive description of the process phases, including the particular stages and the corresponding process parameters, is given in the following text.

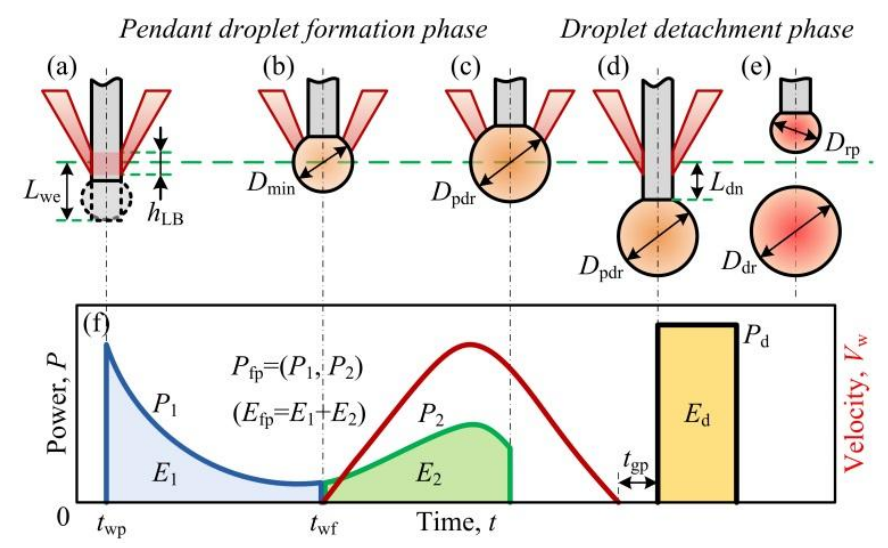

Fig. 3. Process phases and the related main process parameters: (a) Initial wire-end positioning; (b) Formation of a pendant droplet with minimum diameter; (c) Pendant droplet growth to the required diameter; (d) Pendant droplet neck positioning; (e) Pendant droplet detachment; (f) Laser pulse power $P(t)$ and wire feeding velocity $V_{\mathrm{w}}(t)$. 


\subsection{The pendant droplet formation phase}

To form a pendant droplet, the wire-end has to be first properly positioned to the distance $L_{\mathrm{we}}$ with respect to the annular laser beam focus Fig. 3(a). The distance $L_{\mathrm{we}}$ is defined as the distance between the middle point of the laser spot of height $h_{\mathrm{LB}}$ on the wire surface and the lower edge of the wire-end. After positioning of the wire-end, the laser pulse $P_{\mathrm{fp}}(t)=\left(\mathrm{P}_{1}, \mathrm{P}_{2}\right)$ for pendant droplet formation is triggered at time $t_{\mathrm{wp}}$. The laser pulse $P_{\mathrm{fp}}(t)$ is concatenated of two parts $P_{1}$ and $P_{2}$, as is schematically shown in Fig. 3(f) by the blue and green lines. The first part $P_{1}$ of the energy $E_{1}$ is used to melt the wire-end out of which a pendant droplet with a minimum diameter $D_{\mathrm{pdr}}=D_{\min }$ is formed (Fig. 3(b)) under the action of gravity and surface tension forces. Heating of the wire-end material, by means of a simple rectangular laser pulse $P_{1}$ of energy $E_{1}$, initiates oscillation of the pendant droplet during its formation. Therefore, in order to reduce the oscillations of the pendant droplet with min. diameter during its formation, the laser pulse power profile $P_{1}(\mathrm{t})$ has been defined as is shown schematically in Fig. 3(f).

To form a pendant droplet with a larger diameter $D_{\text {pdr }}>D_{\min }$, (Fig. 3(c)) the wire has to be fed with a velocity $V_{\mathrm{w}}(t)$ into the laser beam focus, while at the same time the second part $P_{2}$ of the droplet formation laser pulse of energy $E_{2}$ is applied as shown in Fig. 3(f). The start of the wire feeding at time $t_{\mathrm{wf}}$ is synchronized with the end of the first part $P_{1}$ of the laser pulse. The velocity $V_{\mathrm{w}}(t)$ profile is defined taking into account the desired pendant droplet diameter $D_{\mathrm{pd}}$ and the required distance $L_{\mathrm{dn}}$ for its later detachment. The energy $E_{2}$ of the second part $P_{2}$ of the laser pulse depends on the additional volume of the wire material, which is related to the length $L_{\mathrm{w}}$ of the wire, which has to be fed and melted in order to increase the pendant droplet diameter from $D_{\min }$ up to the required diameter $D_{\text {pdr. }}$. Additionally, to keep the pendant droplet at the laser beam focus during its growth, the velocity of the melting front of the wire which is fed into the laser beam focus should correspond to the wire feeding velocity. Based on this requirement, the power profile $P_{2}(t)$ of the second part of the formation laser pulse of the energy $E_{2}$ is set to be proportional to the wire feeding velocity $V_{\mathrm{w}}(t)$, as is shown schematically in Fig. 3(f).

\subsection{The pendant droplet detachment phase}

In principle, the pendant droplet detachment, which is initiated by a detachment pulse $P_{\mathrm{d}}$, could be started at the end of the droplet formation pulse $P_{\mathrm{fp}}$. However, for reliable pendant droplet detachment by means of the laser detachment pulse $P_{\mathrm{d}}$, the neck of the formed pendant droplet should previously be positioned to an appropriate 
distance $L_{\mathrm{dn}}$ (Fig. 3(d)) with respect to the annular laser beam focus (Kuznetsov et al. 2014). The appropriate distance $L_{\mathrm{dn}}$ between the pendant droplet neck and the annular laser beam focus is achieved by continuing the wire feeding, $V_{\mathrm{w}}(t)$ after the end of the applied droplet formation laser pulse $P_{2}(t)$. In practical cases, the application of the detachment pulse $P_{\mathrm{d}}$, may be additional delayed by a time $t_{\mathrm{gp}}$ due to performance limitations of the pulsed laser source.

As shown schematically in Fig. 3(f), for pendant droplet detachment a high, constant power and short duration detachment pulse $P_{\mathrm{d}}$ of the energy $E_{\mathrm{d}}$ can be applied. It has been shown experimentally (Kuznetsov et al. 2014) that the pendant droplet is detached from the wire-end due to the R-P instability based break-up of the liquid column of molten wire above the pendant droplet neck, which is induced by the annular laser beam pulse. Further it has been shown that the distance $L_{\mathrm{dn}}$ is a very influential parameter since, as well as having an effect on the pendant droplet detachment dynamics, it also influences the diameter $D_{\mathrm{dr}}$ of the detached droplet and the diameter $D_{\mathrm{rp}}$ of the remaining part of the wire-end after pendant droplet detachment, Fig. 3(e)

Based on the presented process description we can summarize that the LDoDG process is governed by the parameters of the annular laser beam pulse $P(t)$ and the wire feeding $V_{\mathrm{w}}(t)$ parameters. Additionally, the pendant droplet formation phase is influenced by the distance $L_{\mathrm{we}}$ of the wire-end with respect to the annular laser beam focus, and the wire-end remaining part diameter $D_{\mathrm{rp}}$ and the corresponding initial temperature $T_{\mathrm{in}}$. The pendant detachment phase of the LDoDG process is additionally influenced by the distance $L_{\mathrm{dn}}$ of the pendant droplet neck with respect to the annular laser beam focus position.

\section{Process analysis and characterization}

In this section a detailed experimental analysis and characterization of the LDoDG process is presented. For this purpose, IR camera based visualization of the LDoDG process is first presented. Then, besides an analysis of the influence of the main process parameters on the process characteristics, the correlations between the influential process parameters specific to particular process phases and their outcomes are considered. In general the LDoDG process does not qualitatively depend on the used wire diameter ranging from 0.25 to $1 \mathrm{~mm}$. Therefore, in the presented experiments and the results of the process characterization a commercially pure $99.6 \% \mathrm{Ni}$ wire (Nickel 200) of diameter $D_{\mathrm{w}}=0.6 \mathrm{~mm}$ was used as a characteristic example. At the end of this section the results of an analysis of the time duration $t_{\mathrm{D}}$ of the process of single DoD generation are presented as an important process characteristic for applications where the frequency of DoD generation plays an important role. 


\subsection{IR camera visualization of the $L D o D G$ process}

An example of the IR camera record of the LDoDG process phases and stages is shown in Fig. 4. In the figure the brighter colour is related to higher temperature of the wire-end and droplet respectively and the horizontal green line denotes the middle point of the laser spot of height $h_{\mathrm{LB}}$ on the wire-end surface. The time $t$ related to the IR images is counted from the trigger time $t=t_{\mathrm{wp}}$ of the pendant droplet formation pulse $P_{1}$ as indicated in Fig. 3(f). In the presented example, a droplet with a diameter of $D_{\mathrm{dr}}=1.05 \mathrm{~mm}$ was generated. The stage corresponding to the minimal diameter $D_{\min }=0.8 \mathrm{~mm}$ pendant droplet formation is presented by the IR image sequence shown in Fig. 4(a). In this figure it can be seen that the wire-end is heated by the laser pulse $P_{1}(t)$ until time $t=7.4 \mathrm{~ms}$, after which the wire-end starts to melt. With further heating within the time interval $t=[7.9$, 8.4] $\mathrm{ms}$ a pendant droplet of minimum diameter $D_{\min }=0.8 \mathrm{~mm}$ is formed from the melted wire-end. In Fig. 4(b) the stage of the pendant droplet growth from the minimum diameter $D_{\min }=0.8$ up to $D_{\text {pdr }}=1.0 \mathrm{~mm}$ by simultaneous application of the laser pulse $P_{2}(t)$ and wire feeding $V_{\mathrm{w}}(t)$ is presented. It can be seen that, due to the proper wire feeding $V_{\mathrm{w}}(t)$, the position of the pendant droplet remains constant during the pendant droplet growth.
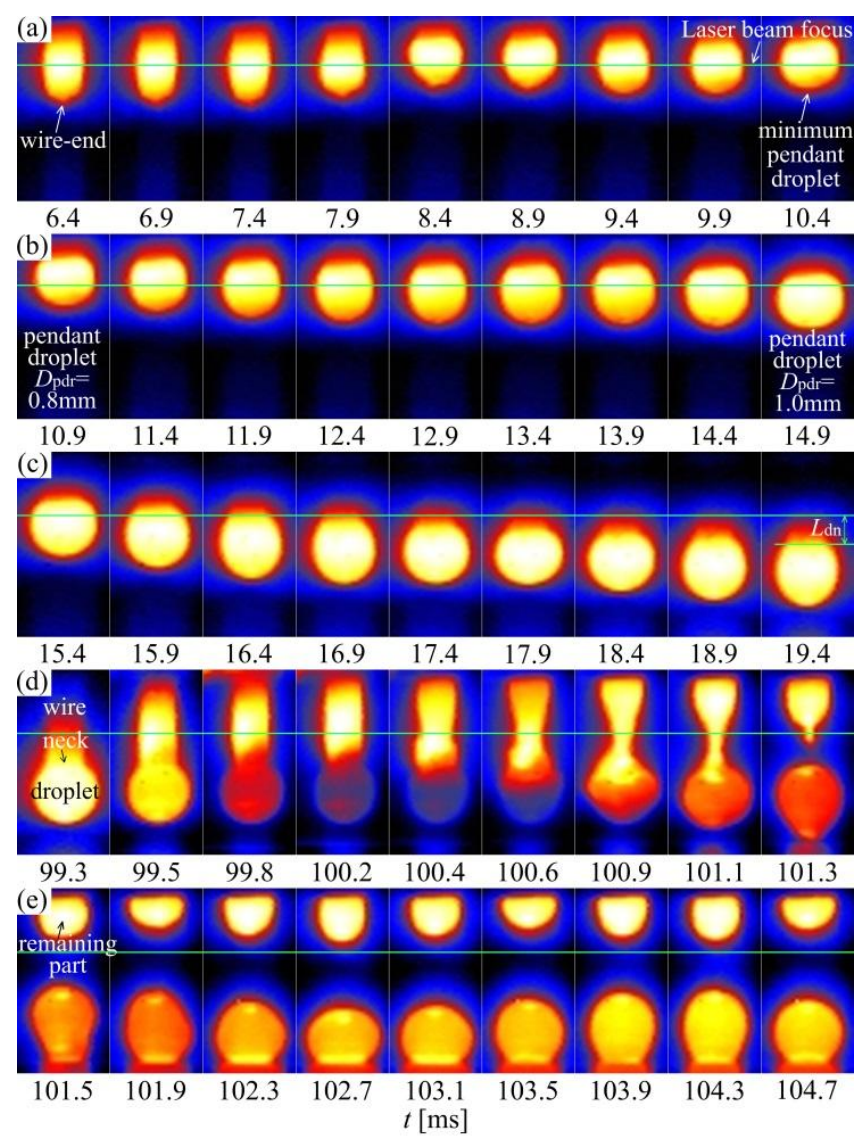
Fig. 4. An example of an IR camera record of the LDoDG process phases and stages : a) Formation of a pendant droplet with minimum diameter; b) Pendant droplet growth up to the required diameter; c) Pendant droplet neck positioning; d) Pendant droplet detachment; e) Droplet deposition.

In Fig. 4(c) part of the stage of the pendant droplet neck positioning onto the distance $L_{\mathrm{dn}}$ with respect to the annular laser beam focus is shown. Successful pendant droplet detachment is then presented in Fig. 4(d). The first image, of the IR sequence of the pendant droplet detachment stage at time $t=99.3 \mathrm{~ms}$, shows the formed pendant droplet positioned at a distance $L_{\mathrm{dn}}=0.6 \mathrm{~mm}$. The detachment laser pulse which heats and melts the wire above the pendant droplet neck is applied at time $t=99.5 \mathrm{~ms}$. At this point a delay time $t_{\mathrm{gp}}=62 \mathrm{~ms}$ due to the performance limitations of the pulsed laser source is taken into account. As can be seen in the following IR images, at the time $t=100.2 \mathrm{~ms}$ a liquid column of the wire above the pendant droplet is obtained, which at time $t=100.4 \mathrm{~ms}$ becomes unstable. Due to the growth of the induced R-P instability of the molten wire column above the pendant droplet neck, the pendent droplet is detached at time $t=101.3 \mathrm{~ms}$. Finally, the IR sequence of the detached droplet deposition on the substrate is shown in Fig 4(e), in which a small vertical oscillation of the deposited droplet can be observed.

\subsection{Pendant droplet formation phase characterization}

The pendant droplet formation process outcome, defined and characterized by the pendant droplet diameter $D_{\text {pdr }}$ and temperature $T_{\mathrm{pdr}}$, is mainly influenced by the laser pulse $P(t)$ and wire-feeding $V_{\mathrm{w}}(t)$ parameters.

Additionally, parameters including the initial wire-end position $L_{\mathrm{we}}$ and the initial wire-end remaining part diameter $D_{\mathrm{rp}}$ and the corresponding temperature $T_{\mathrm{in}}$ have been found to have an important effect. In the following text the performed analysis of the influence of the process parameters on the pendant droplet characteristics is presented, together with the established correlations.

\section{Pendant droplet diameter}

In Fig. 5 a photo of solidified formed pendant droplets of diameter $D_{\text {pdr }}$ in the range from $D_{\min }=0.8$ to $D_{\max }=1.2 \mathrm{~mm}$. In general, the minimum possible droplet diameter $D_{\min }$ depends primarily on the used wire diameter $D_{\mathrm{w}}$ and the height $h_{\mathrm{LB}}$ of the annular laser beam spot on the wire surface. It also depends on the initial state of the wire-end, which can be either a cut wire-end or a wire-end remaining part of diameter $D_{\text {rp. }}$ Taking this into account, the wire-end should be properly positioned at a distance $L_{\text {we }}$ with respect to the laser beam 
focus. Based on theoretical considerations of the annular laser beam focus geometry and the geometry of the wire-end remaining part (Fig. 3(a)), the relations between the minimum pendant droplet diameter $D_{\min }$ and the initial distance $L_{\mathrm{we}}$ have been defined. In Table 1 the calculated initial distance $L_{\mathrm{we}}$ and minimum pendant droplet diameter $D_{\min }$ are shown for the cut wire-end diameter and for the most probable diameters of the wire-end remaining part $D_{\mathrm{rp}}=(0.8,0.85,0.9) \mathrm{mm}$, in the case of droplet generation from a Ni wire of diameter $D_{\mathrm{w}}=0.6 \mathrm{~mm}$, and a height $h_{\mathrm{LB}}=0.8 \mathrm{~mm}$ of the annular laser beam spot. It can be seen that the theoretically possible minimum pendant droplet diameter $D_{\min }$ increases with an increasing diameter of the wire-end remaining part $D_{\mathrm{rp}}$, and, as expected, in the case of larger diameters $D_{\mathrm{rp}}$, smaller distances $L_{\mathrm{we}}$ has to be used to form the minimum pendant droplet.

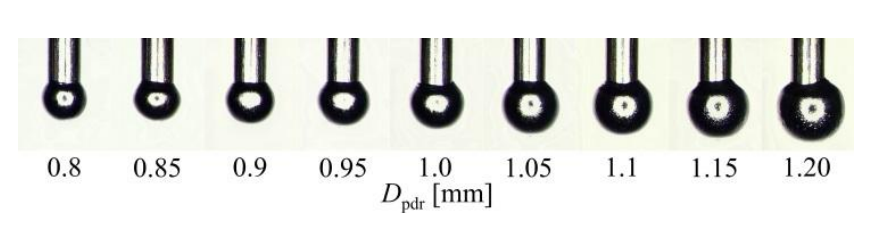

Fig. 5. Examples of solidified pendant droplets with diameters ranging from 0.8 to $1.2 \mathrm{~mm}$.

Table 1. The relationships between $D_{\mathrm{rp}}$ and the corresponding $L_{\mathrm{we}}$, and $D_{\min }$ for $D_{\mathrm{w}}=0.6 \mathrm{~mm}$ and $h_{\mathrm{LB}}=0.8 \mathrm{~mm}$.

\begin{tabular}{|l|l|l|l|l|}
\hline $\boldsymbol{D}_{\text {rp }},[\mathrm{mm}]$ & 0.6 (cut wire-end) & 0.8 & 0.85 & 0.9 \\
\hline $\boldsymbol{L}_{\text {we }},[\mathrm{mm}]$ & 0.6 & 0.51 & 0.49 & 0.46 \\
\hline $\boldsymbol{D}_{\text {min }},[\mathrm{mm}]$ & 0.8 & 0.84 & 0.86 & 0.89 \\
& & & & \\
\hline
\end{tabular}

In order to form a pendant droplet of diameter $D_{\text {pdr }}$ larger than $D_{\min }$, after the formation of the minimum pendant droplet, wire of length $L_{\mathrm{w}}$ is fed into the laser beam focus and melted. Taking into account the volume difference between the required pendant droplet and the existing wire-end remaining part, the correlation between the pendant droplet of diameter $D_{\text {pdr }}$ and the fed wire length $L_{\mathrm{w}}$ can be theoretically defined. In Fig. 6 the theoretical correlations between the fed wire length $L_{\mathrm{w}}$ and the resulting pendant droplet diameter $D_{\mathrm{pdr}}$ are presented by 
means of coloured solid curves, together with the experimental results which are presented by means of different markers for different wire-end remaining part diameters $D_{\mathrm{rp}}$. Around the markers, which denote the median values, the corresponding $3 \sigma$ intervals have been denoted. It can be seen that the experimentally formed minimum pendant droplet diameters are in very good agreement with the theoretical values. It can also be seen that the diameter scatter, which is caused mainly by wire feeding inaccuracy, decreases as the formed pendant droplet diameter $D_{\mathrm{rp}}$ increases. The achieved maximum pendant droplet diameter $D_{\mathrm{pdr}}=1.2 \mathrm{~mm}$ at the given wire diameter $D_{\mathrm{w}}=0.6 \mathrm{~mm}$ is, in our case, limited by the performance of the laser source.

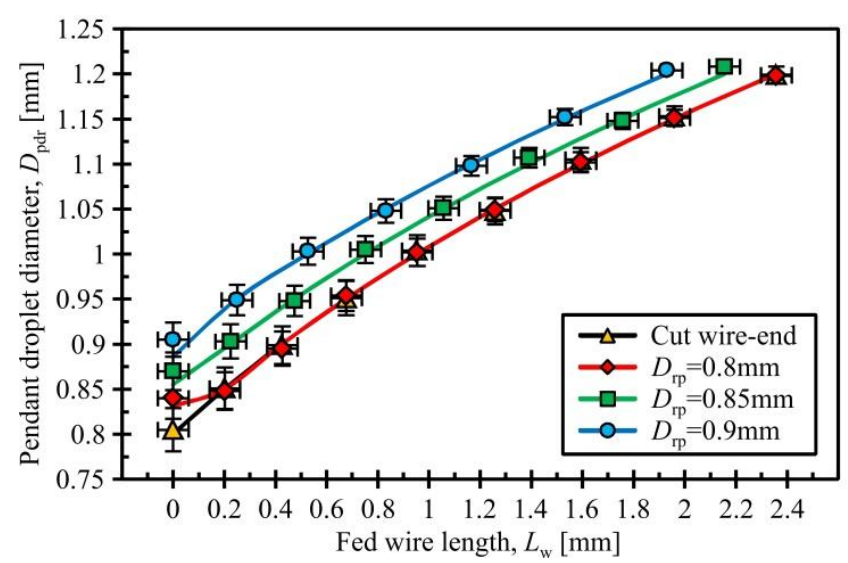

Fig. 6. Theoretical (solid line) and experimental (markers) correlations between the diameter $D_{\text {pdr }}$ of the pendant droplet and the fed wire length $L_{\mathrm{w}}$ to be melted.

The total laser pulse energy $E_{\mathrm{fp}}=E_{1}+E_{2}$ required for the formation of a pendant droplet with a diameter $D_{\mathrm{pdr}}$ was defined experimentally. The energy $E_{1}$ for the minimum pendant droplet formation depends on the wire material, the droplet's diameter $D_{\min }$, and on the initial temperature $T_{\text {in }}$ of the wire-end.. In the case of a Ni wire of diameter $D_{\mathrm{w}}=0.6 \mathrm{~mm}$ and that the wire-end is at room temperature $T_{\text {in }}=20^{\circ} \mathrm{C}$, the energy $E_{1}$ is equals to $11.8 \mathrm{~J}$. The energy $E_{2}$ of the second part of the laser pulse is related to the pendant droplet growth to the desired diameter $D_{\mathrm{pdr}}$, and depends only on the additional wire length $L_{\mathrm{w}}$ which needs to be melted to achieve the diameter $D_{\mathrm{pdr}}$. In Fig. 7 the established correlations between the required total formation laser pulse energy $E_{\mathrm{fp}}$ and the pendant droplet diameter $D_{\text {pdr }}$ median values are shown, together with the corresponding $3 \sigma$ bars, at different initial temperature $T_{\mathrm{in}}$ of the wire-end. It can be seen that the total required energy $E_{\mathrm{fp}}$ is related to the 
pendant droplet diameter $D_{\text {pdr }}$ in a nonlinear, third-power like way, and, as expected, it decreases as the wire-end initial temperature $T_{\text {in }}$ increases.

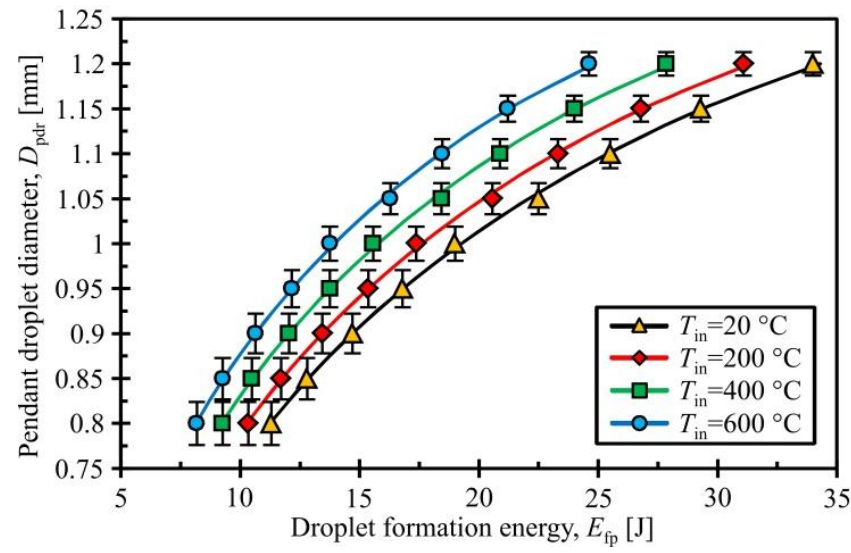

Fig. 7. Relationship between the diameter of the pendant droplet $D_{\mathrm{pdr}}$ and the energy of the formation laser pulse $E_{\text {fp. }}$

\section{Temperature analysis}

The temperature analysis of the pendant droplet formation phase was performed based on the surface temperature $T_{\mathrm{ws}}(t)$ measurement of the wire-end acquired at the spot of the laser beam focus by the two-colour pyrometer. Examples of time dependent graphs of the temperature $T_{\mathrm{ws}}(t)$, acquired during the formation of pendant droplets of diameters $D_{\mathrm{pdr}}=0.8,1.0$, and $1.2 \mathrm{~mm}$, are shown in Fig. 8(a). Additionally, in Fig. 8(b), the corresponding pendant droplet formation laser pulse power $P_{\mathrm{fp}}(t)$ and the wire feeding velocity $V_{\mathrm{w}}(t)$ are shown by solid and dashed lines respectively. In both graphs the time $t$ denotes the elapsed time from the moment of the triggering of the laser pulse $P_{\mathrm{fp}}(t)$.

As can be seen from the temperature $T_{\text {ws }}(t)$ graphs (Fig. 8(a)), the maximum temperature of the wire at the laser beam focus spot remains below the boiling temperature $\left(2730{ }^{\circ} \mathrm{C}\right)$ of the Ni. This means that the pendant droplet formation process phase occurs without unfavourable evaporation of the $\mathrm{Ni}$ wire material. At the beginning of the laser pulse, the temperature $T_{\mathrm{ws}}(t)$ of the wire-end surface rises extremely fast, and reaches a peak value of $T_{\mathrm{ws}} \approx 2200{ }^{\circ} \mathrm{C}$. At the time $t=10 \mathrm{~ms}$, which corresponds to the duration of the first part $P_{1}(t)$ of the laser pulse for pendant droplet formation, the temperature $T_{\mathrm{ws}}$ becomes stabilized around $1500{ }^{\circ} \mathrm{C}$. The observed 
temperature peak is presumably caused by a fast decrease of the wire surface absorptivity from an initially high absorptivity related to a rough surface to a low absorptivity related to a shiny melted surface.

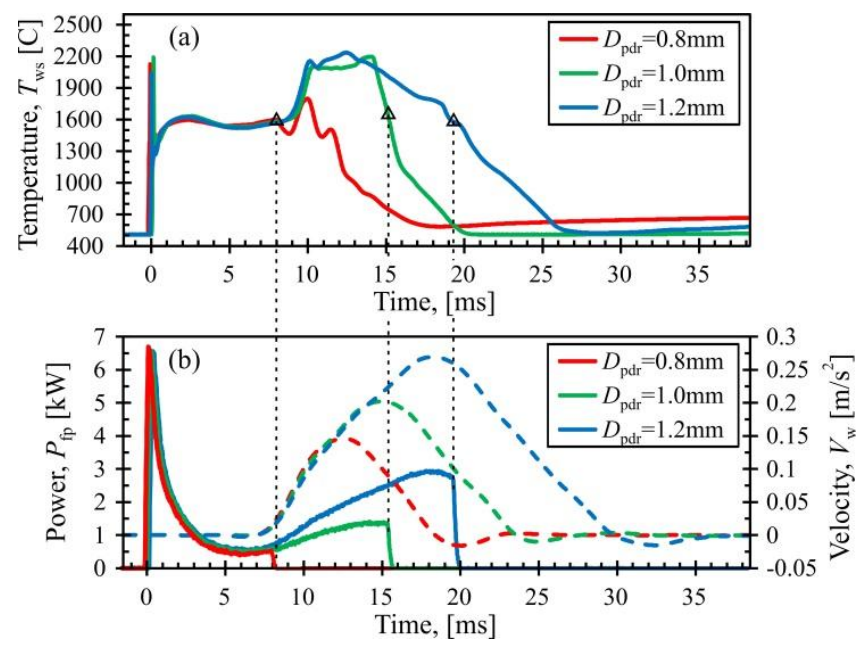

Fig. 8. a) Surface temperature $T_{\mathrm{ws}}$ of the wire-end during the pendant droplet formation phase, b) the corresponding process parameters $P_{\mathrm{fp}}(t)$ (solid line) and $V_{\mathrm{w}}(t)$ (dashed line).

In the case of pendant droplet formation of minimum diameter $\left(D_{\mathrm{pdr}}=0.8 \mathrm{~mm}\right.$, the solid red line in Fig. 8(b)) at time $t=10 \mathrm{~ms}$, the laser power $P_{\mathrm{fp}}(t)$ is set to zero and wire feeding $V_{\mathrm{w}}(t)$ (the red dashed line) is applied so that the pendant droplet is at the proper position $L_{\mathrm{dn}}$ with respect to the annular laser beam focus for its detachment. Thus, after time $t=10 \mathrm{~ms}$, the acquired surface temperature, which corresponds to the temperature of the wire above the pendant droplet neck, decreases to $T_{\text {ws }} \approx 700^{\circ} \mathrm{C}$.

In the case of the pendant droplet formation of larger diameters $\left(D_{\mathrm{pdr}}>D_{\mathrm{min}}\right)$, at $t=10 \mathrm{~ms}$ the pendant droplet growth from the minimum value to the desired droplet diameters (the green and blue lines in Fig. 8(b)) is started by the simultaneous application of the wire feeding $V_{\mathrm{w}}(t)$ (the dashed lines) and the second part $P_{2}(t)$ (the solid lines) of the laser pulse $P_{f p}(t)$. It can be seen (the green and blue lines in Fig. 8(a)) that, after time $t=10 \mathrm{~ms}$ the pendant droplet temperature $T_{\mathrm{ws}}(t)$ firstly rises to about $2200^{\circ} \mathrm{C}$. The temperature $T_{\mathrm{ws}}(t)$ fluctuations observed within the time interval $t=(10,18) \mathrm{ms}$ are related to the oscillations of the pendant droplet. Further wire feeding (the green and blue dashed lines in Fig. 8(b)), which occurs after the end of the pendant droplet formation laser pulse $P_{f p}(t)$, is used to ensure the proper position $L_{\mathrm{dn}}$ of the pendant droplet neck with respect to the laser beam 
focus. Thus the following measured temperature $T_{\mathrm{ws}}(t)$ (the green and blue solid lines in Fig. 8(a)), corresponds to the temperature of the wire above the pendant droplet neck, which quickly drops and settles around $500{ }^{\circ} \mathrm{C}$. Based on this, as an estimate of the temperature of the formed pendant droplet $T_{\mathrm{pdr}}$, the temperature $T_{\mathrm{ws}}(t)$ reached at the end of the formation laser pulse, denoted in the Fig. 8(a) by triangles, can be used. It can be seen that the corresponding pendant droplet temperatures $T_{\mathrm{pdr}}$ stay within the range $1600-1700^{\circ} \mathrm{C}$, i.e. $145-245^{\circ} \mathrm{C}$ above the melting point $\left(1455^{\circ} \mathrm{C}\right)$ of the used $\mathrm{Ni}$ wire.

\subsection{Droplet detachment phase characterization}

Pendant droplet detachment is the most critical phase of LDoDG process since it affects the process stability and lateral scatter of detached droplets. Within this context, successful pendant droplet detachment means that the pendant droplet is detached without molten splashes, i.e. satellite droplets of molten material, and that the detached droplet has the desired characteristics including its diameter $D_{\mathrm{dr}}$, temperature $T_{\mathrm{dr}}$, and a low lateral scatter. Beside the parameters of the detachment pulse $P_{\mathrm{d}}$, the annular laser beam focus distance $L_{\mathrm{dn}}$ from the pendant droplet neck has been shown to be the most influential parameter of the droplet detachment phase. In the following the results of the analysis of the latter's influence on the droplet detachment regime, the detached droplet diameter $D_{\mathrm{dr}}$, and temperature $T_{\mathrm{dr}}$, the wire-end remaining part diameter $D_{\mathrm{rp}}$, and the lateral scatter of the detached droplets, are presented. Additionally, the correlations between the detached droplet diameter $D_{\mathrm{dr}}$ and the corresponding droplet temperature $T_{\mathrm{dr}}$ are presented.

\section{Detachment droplet regimes}

In the case of the annular laser beam, a pendant droplet is detached based on the R-P instability of the molten wire column above the pendant droplet neck. For this purpose, in general a high power and short duration detachment pulse $P_{\mathrm{d}}$ has to be applied. To detach a Ni pendant droplet from a wire of diameter $D_{\mathrm{w}}=0.6 \mathrm{~mm}$, a simple rectangular detachment pulse of power $P_{\mathrm{d}}=8 \mathrm{~kW}$, and duration $t_{\mathrm{d}}=1.4 \mathrm{~ms}$, with corresponding energy $E_{\mathrm{d}}=12.8 \mathrm{~J}$, was used.

As presented in (Kuznetsov et al. 2014), in the case of the defined detachment pulse $P_{\mathrm{d}}$ parameters, different droplet detachment regimes, including detachments with droplet splashes, successful detachment, droplet detachment with reattachment, and non-detachment of pendant droplet, have been observed, depending on the distance $L_{\mathrm{dn}}$. 
To characterize the influence of the distance $L_{\mathrm{dn}}$ on pendant droplet detachment, the initial vertical velocity $V_{\mathrm{v}, \mathrm{dr}}$ of the detached droplets was estimated from the IR images. For the selected pendant droplet diameters $D_{\text {pdr }}$ the median values and standard deviation bars of the initial velocity $V_{\mathrm{z}, \mathrm{dr}}$ of the detached droplets are shown, in dependence on the distance $L_{\mathrm{dn}}$, in Fig. 9. Additionally, in Fig. 9 the corresponding detachment regimes observed from the IR records are marked. It can be seen that successful and stable droplet detachment, in the case of a $\mathrm{Ni}$ wire with a diameter $D_{\mathrm{w}}=0.6 \mathrm{~mm}$, can be achieved with distances $L_{\mathrm{dn}}$ ranging from 0.3 to $1.0 \mathrm{~mm}$. At smaller distances $L_{\mathrm{dn}} \leq 0.2 \mathrm{~mm}$, pendant droplet detachment is accompanied by splashes, whereas at larger distances $L_{\mathrm{dn}} \geq 1.0 \mathrm{~mm}$ pendant droplet re-attachment and non-detachment take place. The initial vertical velocity $V_{\mathrm{z}, \mathrm{dr}}$ of the detached droplets decreases within the range from 0.4 to $0.0 \mathrm{~m} / \mathrm{s}$ with increasing $L_{\mathrm{dn}}$. Within the region of reattached and non-detached droplets $\left(L_{\mathrm{dn}} \geq 1.0 \mathrm{~mm}\right)$ the initial velocities $V_{\mathrm{z}, \mathrm{dr}}$ are negative or zero. It is also evident from Fig. 9 that, for successful droplet detachment, the initial vertical velocity $V_{\mathrm{z}, \mathrm{dr}}$ increases with increased values of $D_{\mathrm{pdr}}$.

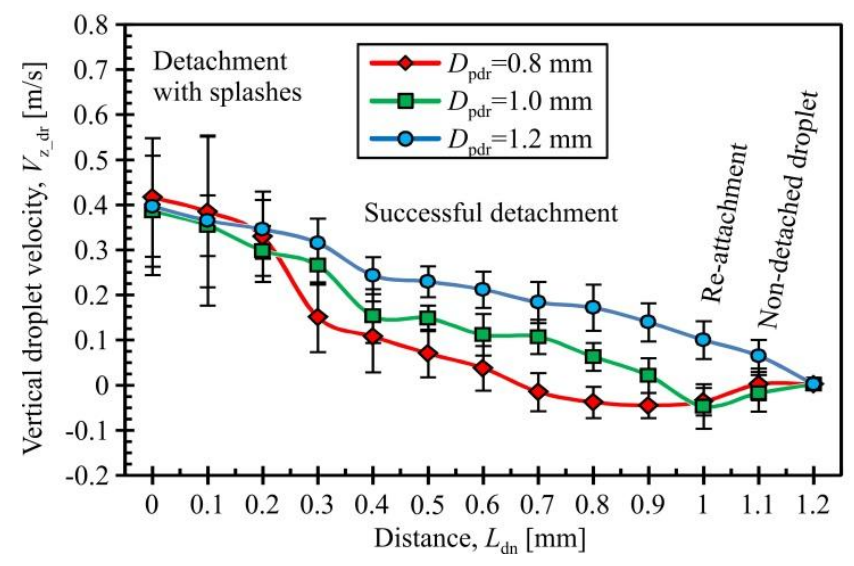

Fig. 9. Droplet detachment regimes and the initial vertical velocity $V_{\mathrm{v}, \mathrm{dr}}$ of the detached droplets vs. the distance $L_{\mathrm{dn}}$

Diameter of the detached droplet

At the moment of detachment of the pendant droplet due to R-P instability based break-up of the molten column of the wire above the pendant droplet neck, the volume of the molten column, which depends on the height $h_{\mathrm{LB}}$ of the annular laser beam on the wire surface, and on the distance $L_{\mathrm{dn}}$, splits into two parts. The upper part 
remains attached as a wire-end remaining part of diameter $D_{\mathrm{rp}}$, while the lower part joins the volume of the detached droplet.

In Fig. 10 the experimentally obtained dependence of the diameter $D_{\mathrm{rp}}$ (dashed lines) of the wire-end remaining part and the detached droplet diameter $D_{\mathrm{dr}}$ (solid lines) on the distance $L_{\mathrm{dn}}$ are shown for different pendant droplet diameters $D_{\mathrm{pdr}}$. The markers denote the median values, and the bars denote the $3 \sigma$ deviation. It can be seen that independently of the pendant droplet diameter $D_{\mathrm{pdr}}$, the wire-end remaining part diameter $D_{\mathrm{rp}}$ slightly decreases within the range from 0.9 to $0.8 \mathrm{~mm}$ as the distance $L_{\mathrm{dn}}$ is increased. The detached droplet diameter $D_{\mathrm{dr}}$ therefore slightly increases with the distance $L_{\mathrm{dn}}$. In the case of larger pendant droplet diameters $\left(D_{\mathrm{pdr}}=1.0\right.$ and $1.2 \mathrm{~mm}$ ) the detached droplet diameter $D_{\mathrm{dr}}$ is also larger than the diameter of the pendent droplet for all distances $L_{\mathrm{dn}}$. However, in the case of the minimum pendant droplet $\left(D_{\mathrm{pdr}}=0.8 \mathrm{~mm}\right)$, at shorter distances $L_{\mathrm{dn}}<0.3 \mathrm{~mm}$ the detached droplet diameter $D_{\mathrm{dr}}$ is smaller, whereas with further increasing of $L_{\mathrm{dn}}$ the detached droplets are larger than the preceding pendant droplets. The reason for this experimentally observed particularity could be sought for in the geometrical relations between the annular laser beam spot height $h_{\mathrm{LB}}$, and the pendant droplet diameter $D_{\text {pdr }}$ (Fig. 3b). Namely, in the case of the minimal droplet diameter a much larger portion of the pendant droplet surface and at a different angle is illuminated by the laser beam than in the case of larger pendant droplets, which influences the annular laser beam - pendant droplet interaction and presumably leads to the observed qualitative differences. As shown in Fig. 10, the performed experiments in the regime of successful detachment, with a Ni wire of $0.6 \mathrm{~mm}$ diameter, indicate high repeatability of the detached droplet diameter $D_{\mathrm{dr}}$ ranging from 0.85 to $1.25 \mathrm{~mm}$, with a corresponding estimated standard deviation of $15 \mu \mathrm{m}$.




Fig. 10. Dependence of the remaining part diameter $D_{\mathrm{rp}}$ and the detached droplet diameter $D_{\mathrm{dr}}$ on the distance $L_{\mathrm{dn}}$.

\section{Temperature of the detached droplet}

Beside the droplet diameter $D_{\mathrm{dr}}$, the droplet temperature $T_{\mathrm{dr}}$ is an important characteristic for droplet based applications. Temperature analysis of the droplet detachment phase and characterization of the corresponding detached droplet temperature $T_{\mathrm{dr}}$ was performed based on the signals acquired by the two- colour and singlecolour pyrometers. In the case of the two-colour pyrometer, the surface temperature $T_{\text {ws }}(t)$ of the wire at the laser beam focus spot, which was at a distance $L_{\mathrm{dn}}$ above the pendant droplet neck, was acquired, whereas in the case of the single-colour pyrometer, the surface temperature $T_{\mathrm{dr}}$ of the detached droplet was acquired at the moment of the pendant droplet detachment at a droplet equator position.

The variation over time of the wire surface temperatures $T_{\mathrm{ws}}(t)$ for three different pendant droplet diameters $D_{\mathrm{pdr}}$ is shown in Fig. 11(a). In the Fig. 11(b) the corresponding measured rectangular laser pulse power profile is shown. The observed peak temperature values, around $2400^{\circ} \mathrm{C}$, are related to the laser pulse and do not exceed the boiling point $\left(2730^{\circ} \mathrm{C}\right)$ of the Ni wire. The oscillating decay of the temperature $T_{\mathrm{ws}}(t)$ that follows after the peak value corresponds to the cooling down and mechanical oscillations of the wire-end remaining part after pendant droplet detachment. The surface temperature $T_{\mathrm{dr}}$ of the detached droplets versus the droplet diameter $D_{\mathrm{dr}}$ within the range from 0.85 to $1.25 \mathrm{~mm}$ is shown in Fig. 12. In the graph the median values of $T_{\mathrm{dr}}$ with the corresponding $3 \sigma$ deviation bars are marked, from which a slight increase in the droplet temperature $T_{\mathrm{dr}}$ with increasing droplet diameter $D_{\mathrm{dr}}$ is evident. It can be seen (Fig. 12) that the temperature of the detached droplets remains $200-300{ }^{\circ} \mathrm{C}$ above the melting point of the $\mathrm{Ni}$ wire. 

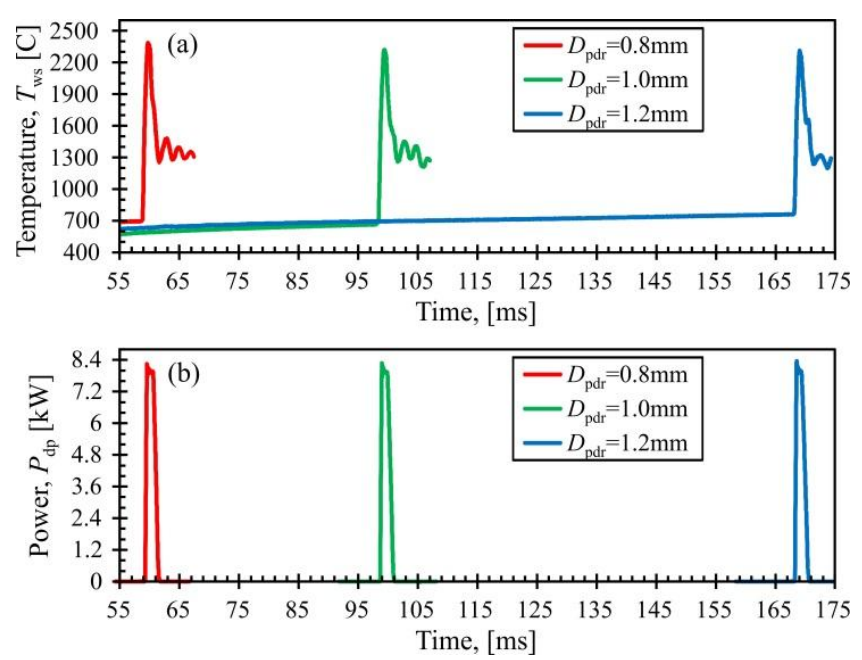

Fig. 11. a) Temperatures $T_{\text {ws }}$ of the wire material at the laser beam focus spot during the droplet detachment phase, b) The detachment laser pulse $P_{\mathrm{dp}}$ power profiles.

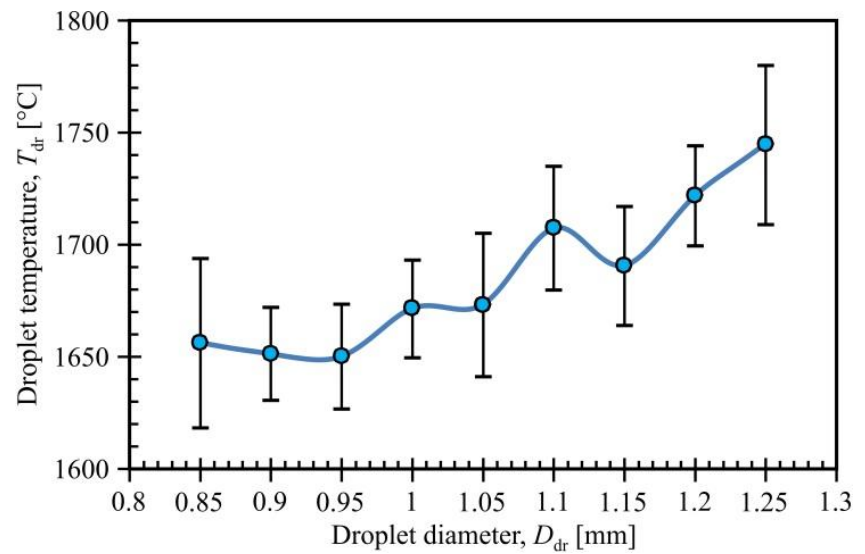

Fig. 12. Droplet temperature $T_{\mathrm{dr}}$ vs. droplet diameter $D_{\mathrm{dr}}$.

\section{Scatter of the detached droplet position}

For droplet based applications, lateral scatter of the position of the detached droplets is of high importance since it affects droplet deposition accuracy. This scatter was characterised based on the analysis of the detached droplets' centroid trajectories extracted from the IR records of the DoD generation process. In Fig. 13 the dependence of detached droplet centroid lateral position distance $Y_{\mathrm{dr}}$ from the wire axis versus the distance $L_{\mathrm{dn}}$ at a selected stand-off distance $L_{\mathrm{ds}}=3.0 \mathrm{~mm}$ is presented. As can be seen from this figure, in the case of a pendant 
droplet diameter $D_{\mathrm{pdr}}=0.8 \mathrm{~mm}$ a relatively large deviation of the droplet position was observed over the interval of distances $L_{\mathrm{dn}}=(0.3,1.0) \mathrm{mm}$, providing successful detachment. In the case of larger pendant droplets $D_{\text {pdr }}=1.0$ and $1.2 \mathrm{~mm}$ the detachment results in a much smaller lateral scatter, which can be further decreased by decreasing the stand-off distance $d_{\mathrm{s}}$ and by optimisation of the detachment pulse. Similarly, as in the case of the above-mentioned experimentally observed particularity related to detached droplet diameter $D_{\mathrm{dr}}$ dependency on the distance $L_{\mathrm{dn}}$ at the minimum sized pendant droplet (see Fig. 10), the reason for the experimentally observed larger lateral deviation of the droplet position $Y_{\mathrm{dr}}$ in the case of minimal diameter droplet generation at shorter distances $L_{\mathrm{dn}}$ could be sought for in the geometrical relations and related interactions between the annular laser beam spot height $h_{\mathrm{LB}}$, and the pendant droplet diameter $D_{\mathrm{pdr}}$. Based on this it can be concluded that small diameter droplets, instead of being generated from a pendant droplet of minimal diameter, should be generated from a wire of smaller diameter with the aim of achieving a lower lateral scatter of the detached droplet position $Y_{\mathrm{dr}}$.

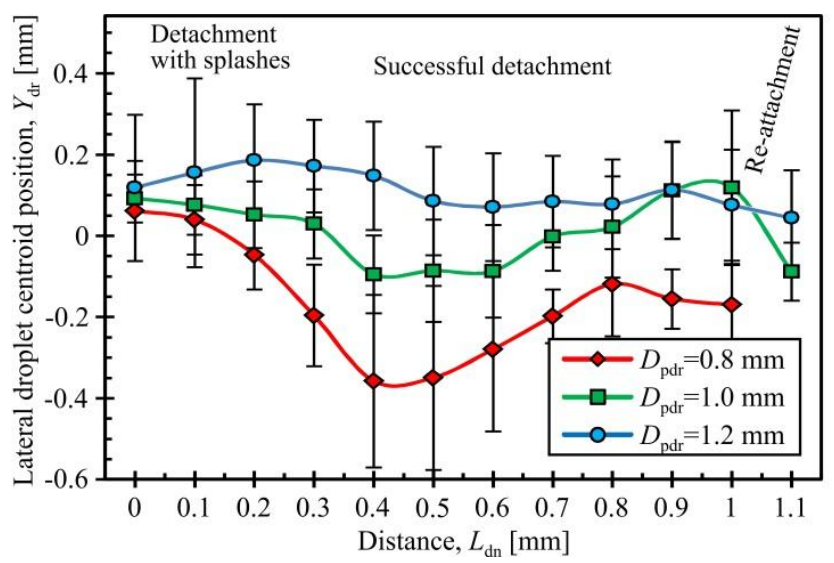

Fig. 13. Lateral droplet centre position of detached droplets at a stand-off distance $d_{\mathrm{s}}=3.0 \mathrm{~mm}$ versus the distance $L_{\mathrm{dn}}$.

\subsection{Laser DoD generation process time duration}

For certain metal droplet based applications, besides the droplet characteristics including droplet diameter $D_{\mathrm{dr}}$ and temperature $T_{\mathrm{dr}}$ and lateral scatter, the corresponding process time duration $t_{\mathrm{dg}}$ i.e. the time required for single DoD generation, is of high importance. The time $t_{\mathrm{dg}}$ is especially important for applications where the 
frequency of DoD generation plays an important role. The duration time $t_{\mathrm{dg}}$ of the LDoDG process was defined by the time at the end of the detachment pulse $P_{\mathrm{d}}$. The duration time $t_{\mathrm{dg}}$ depends, in general, on the wire material and diameter $d_{\mathrm{w}}$, and on the generated droplet diameter $D_{\mathrm{dr}}$, and therefore includes the time $t_{\mathrm{wp}}$ of the wire-end initial positioning to the distance $L_{\mathrm{we}}$ with respect to the annular laser beam focus, and the time $t_{\mathrm{i}}$ required for pendant droplet formation and detachment. Ideally the detachment laser pulse should be applied directly after the pendant droplet neck is positioned to the distance $L_{\mathrm{dn}}$ with respect to the laser beam focus. The corresponding ideal time $t_{\mathrm{i}}$ is defined by the sum of the duration of the pendant droplet formation pulse, the time required for the pendant droplet neck positioning, and the duration of the droplet detachment pulse. However, in real cases $t_{\mathrm{i}}$ can be increased by some additional time $t_{\mathrm{gp}}$ which is required due to the performance limits of the laser source, so that the corresponding LDoDG process duration can be defined by the expression: $t_{\mathrm{dg}}=t_{\mathrm{wp}}+t_{\mathrm{i}}+t_{\mathrm{gp}}$.

In Fig. 14 the characteristic times $t_{\mathrm{wp}}, t_{\mathrm{i}}$, and $t_{\mathrm{gp}}$ of LDoDG are shown versus the droplet diameter $D_{\mathrm{dr}}$ generated from $\mathrm{Ni}$ wire of $0.6 \mathrm{~mm}$ diameter. Experimental median values with corresponding $3 \sigma$ deviations of the time $t_{\mathrm{wp}}$ are shown by yellow triangles and a black curve. As expected, the time $t_{\mathrm{wp}}$ does not depend on the droplet diameter $D_{\mathrm{dr}}$. The corresponding experimentally estimated ideal time $t_{\mathrm{i}}$ versus droplet diameter $D_{\mathrm{dr}}$ is denoted by red diamonds. However, in the described case the applied laser source can produce laser pulses with a limited maximum averaged power of $250 \mathrm{~W}$, which means the laser needs a certain time $t_{\mathrm{gp}}$ for garnet pumping between the end of the pendant droplet neck positioning and the onset of the droplet detachment pulse $P_{\mathrm{d}}$, (Fig. 3(f)). The estimated time $t_{\mathrm{gp}}$ is directly related to the generated droplet diameter $D_{\mathrm{dr}}$ as denoted by the green squares (Fig. 14). In accordance with the graphs presented in Fig. 14, in the case of laser DoD generation from a Ni wire of diameter $D_{\mathrm{w}}=0.6 \mathrm{~mm}$, the total time $t_{\mathrm{t}}$ lies within the interval $[180,280] \mathrm{ms}$. The corresponding maximum frequency of laser DoD generation, which depends on the generated droplet diameter $D_{\mathrm{dr}}$, therefore lies, in our case, within the interval $[3.5,5.5] \mathrm{Hz}$. 


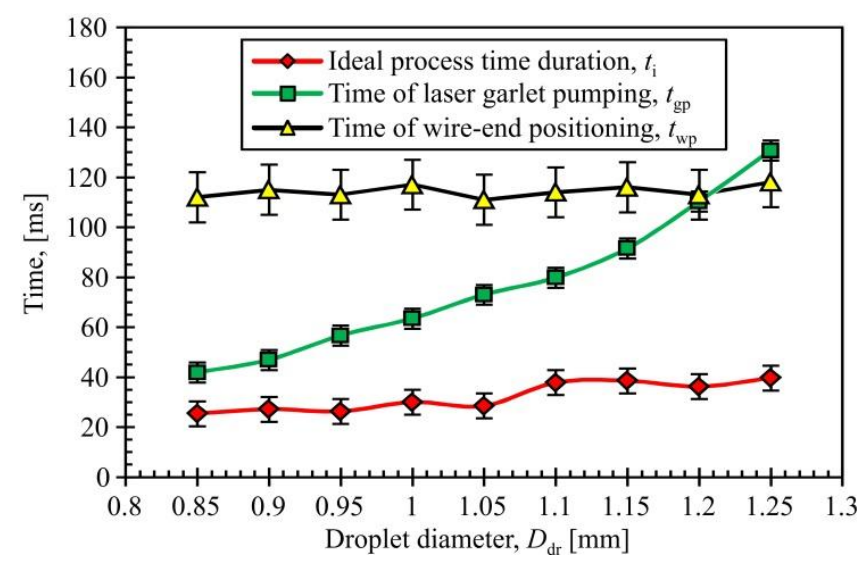

Fig. 14. Characteristic times of the LDoDG process duration vs. droplet diameter $D_{\mathrm{dr}}$.

\section{Examples of deposited droplets}

With the aim of demonstrating the capabilities of the novel annular LDoDG process for possible metal droplet based industrial applications, some examples of droplets deposited onto a metal surface are shown in the following. In the droplet deposition experiments, droplets generated from a Ni wire of diameter $D_{\mathrm{w}}=0.6 \mathrm{~mm}$ were deposited on the surface of a Ti substrate of $0.4 \mathrm{~mm}$ thickness. The droplets were deposited without preliminary preheating of the substrate surface, and Argon was used as a shielding gas. The stand-off distance $L_{d s}$ of the pendant droplet from the substrate surface was $L_{d s}=1.0 \mathrm{~mm}$. To qualitatively demonstrate the control of the generated droplet of diameter $D_{\mathrm{dr}}$, as the first example, in Fig. 15 top and side views of a set of deposited Ni droplets with increasing diameters ranging from 0.85 to $1.25 \mathrm{~mm}$, with a diameter step of $50 \mu \mathrm{m}$, are shown. Although influenced by many parameters, which remains to be quantitatively characterized in detail, from the enlarged details of the three selected droplets of diameters $D_{\mathrm{dr}}=0.85,1.05$ and $1.25 \mathrm{~mm}$ it can be observed that the droplet substrate surface contact angle $\alpha$ slightly increases with an increase in the droplet diameter $D_{\mathrm{dr}}$ and higher internal energy, respectively. 


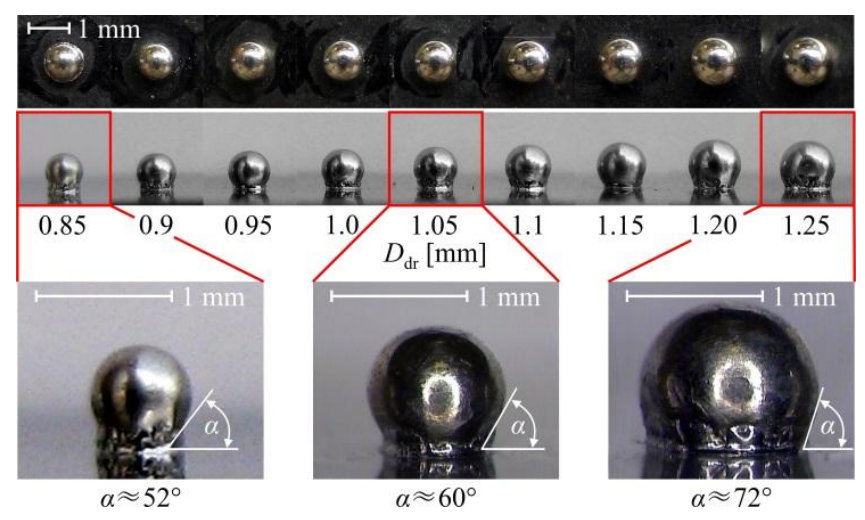

Fig. 15. Examples of deposited droplets with increasing diameter $D_{\mathrm{dr}}$, ranging from 0.85 to $1.25 \mathrm{~mm}$ and selected enlarged droplets.

To qualitatively demonstrate the repeatability of the generated droplet diameter $D_{\mathrm{dr}}$ and the lateral scatter of droplet positioning, top and side views of a set of twelve droplets, with predefined droplet diameters of $D_{\mathrm{dr}}=0.85,1.05$ and $1.25 \mathrm{~mm}$, deposited in a straight line, are shown in Fig. 16. In addition to these droplets which were deposited in a straight line, droplets were also deposited in the simple pattern of a square matrix, and in more complex patterns such as plain text and a snowflake, as shown in Fig. 17. In the presented examples three different droplet diameters $D_{\mathrm{dr}}=0.85,1.05$ and $1.25 \mathrm{~mm}$ were used.

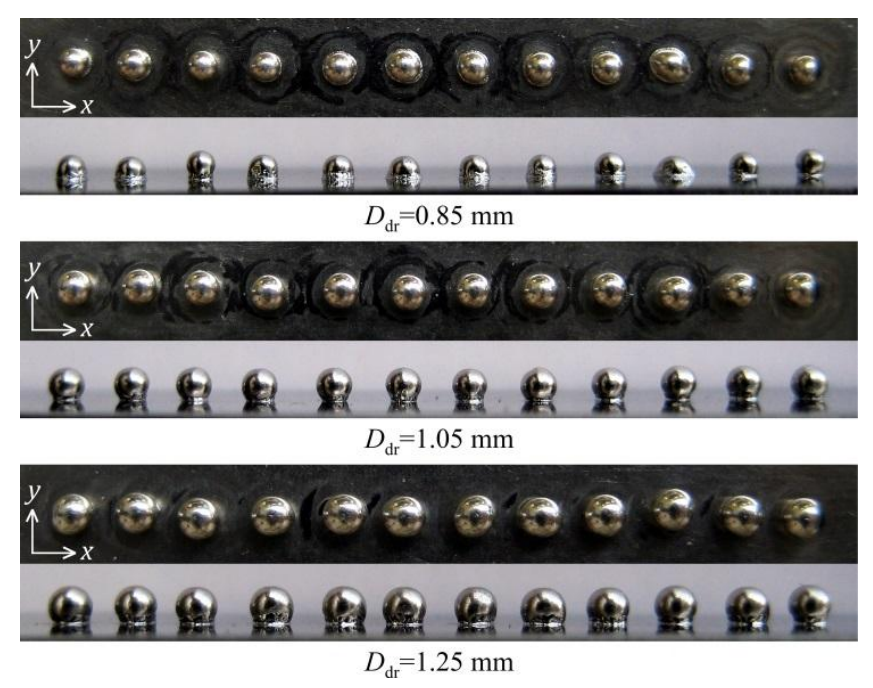

Fig. 16. Examples of top and side views of in line deposited droplets with diameters of $D_{\mathrm{dr}}=0.85,1.05$, and $1.25 \mathrm{~mm}$. 


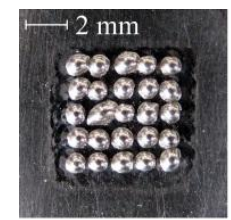

$D_{\mathrm{dr}}=0.85 \mathrm{~mm}$

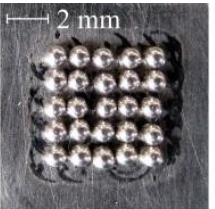

$D_{\mathrm{dr}}=1.05 \mathrm{~mm}$

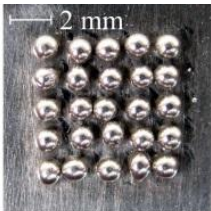

$D_{\mathrm{dr}}=1.25 \mathrm{~mm}$

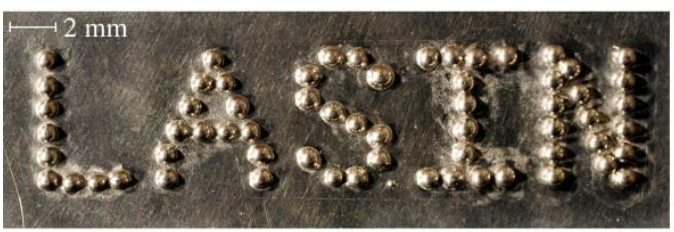

Acronym "Laboratory of Synergetics" Droplet diameter: $D_{\mathrm{dr}}=1.05 \mathrm{~mm}$

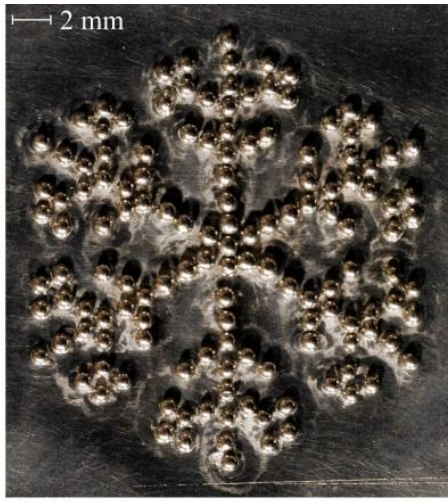

Snowflake $D_{\mathrm{dr}}=0.85 ; 0.95 ; 1.05 \mathrm{~mm}$

Fig. 17. Examples of various 2D patterns created by the deposition of droplets with different diameters $D_{\mathrm{dr}}$

The main potential application of the A-LDG process is droplet base micro joining, which has already been confirmed by the joining of temperature sensitive components, the joining of dissimilar components, and the joining of wire with thin metallization (Govekar et al., 2009). The presented simple 2D patterns indicate a potential application also in the fabrication of 3D micro structures. In the case of 3D structuring, the A-LDG process could be compared to the TIG arc micro welding based dispositioning of micro beads (Terakubo et al., 2005), which has been shown to be very well suited to the fabrication of $3 \mathrm{D}$ metal objects. The advantages and disadvantages of the A-LDG process depend on its application, and stem from the use of the laser beam as an energy source for heating. A high level of control of the laser input energy, a low heat affected zone, and low thermal loads of the surroundings are of high importance when realizing droplet based micro joints of temperature sensitive components in the electronics industry. Additionally the coaxiality of the laser beam and wire enables pre-heating of the deposited spot by the annular laser beam, which can be additionally used to control the process of micro joining or 3D structuring.

\section{Conclusions}

In this paper the proposed novel annular laser droplet generation system (A-LDG) and process for laser drop-ondemand generation (LDoDG) from a metal wire have been presented. The main component of the system is the designed A-LDG head by means of which a Gaussian laser beam is shaped into an annular laser beam, which is guided coaxially with the wire axis and focused on the circumference of the wire-end. 
The related LDoDG process consists of two sequential phases: pendant droplet formation, and pendant droplet detachment from the wire-end. The properties of the annular laser beam, in the phase of pendant droplet formation, enable uniform heating of the wire-end around its circumference and formation of a pendant droplet by means of a laser pulse. The formed pendant droplet is then detached by higher power laser pulse induced Rayleigh-Plateau (R-P) instability of the molten column of wire above the pendant droplet neck.

The main process parameters which influence both phases are the laser pulse power $P(t)$ and the wire feeding $V_{\mathrm{w}}(t)$ parameters. The diameter $D_{\mathrm{pdr}}$ of the pendant droplet and the related temperature $T_{\mathrm{pdr}}$ are influenced by the energy of the pendant droplet formation pulse and by the wire feeding parameters. Additionally, the pendant droplet diameter and the related laser pulse and wire feeding parameters are influenced by the initial wire-end remaining part diameter $D_{\text {rp }}$, the temperature $T_{\text {in }}$, and the initial positioning $L_{\mathrm{we}}$ of the wire-end with respect to the annular laser beam focus. It has been show that, the pendant droplet detachment phase, besides the energy $E_{\mathrm{d}}$ of a high power detachment pulse $P_{\mathrm{d}}$, is governed by the distance $L_{\mathrm{dn}}$ of the pendant droplet neck with respect to the annular laser beam focus. The distance $L_{\mathrm{dn}}$ influences the pendant droplet detachment dynamics, and related detached droplet diameter $D_{\mathrm{dr}}$, the initial velocity $V_{\mathrm{z}, \mathrm{dr}}$, and the lateral scatter $Y_{\mathrm{dr}}$.

The main advantage of the proposed A-LDG system and the LDoDG process is flexibility from the point of view of the wire material and diameter that can be used for DoD generation. The application of a laser beam for wire heating means that droplets of high melting point can be generated at low thermal loads of the surroundings. By means of precise control of the laser pulse and wire feeding parameters, and due to the R-P based pendant droplet detachment, from a wire with certain diameter, droplets of different and predefined diameters can be generated with high repeatability, with the minimum quantity of droplet splashes and a low lateral scatter of the detached droplets. Additionally, the annular laser beam caustic enables preheating of the droplet deposition spot, which widens the possible applications of DoD generated droplets.

As presented, droplets of various diameters $D_{\mathrm{dr}}$, ranging from 0.85 to $1.25 \mathrm{~mm}$, with a diameter resolution of $50 \mu \mathrm{m}$ and a standard deviation of $15 \mu \mathrm{m}$, can be generated from a wire made from commercially pure $99.6 \% \mathrm{Ni}$ (Nickel 200), with a diameter of $0.6 \mathrm{~mm}$. The corresponding droplet temperature $T_{\mathrm{dr}}$ in the range from 1650 to $1740{ }^{\circ} \mathrm{C}$, which is $195-295^{\circ} \mathrm{C}$ above the melting point of the Ni material, can be achieved. A relative low lateral scatter of the detached droplet position $Y_{\mathrm{dr}}$ was observed, which additionally could be decreased by the detachment pulse optimization. 
Although demonstrated by simple patterns of Ni droplets deposited on a Ti substrate only, in summary it can be concluded that the designed A-LDG head, together with the presented A-LDG system and the process of DoD generation from a metal wire, are very promising for metal droplet based applications (micro joining and 3D structuring) where droplets of precise diameter and high temperature need to be generated with low thermal loads of the surroundings. The presented A-LDG system and LDoDG process can also be used also for DoD sequence generation. However, in the case of DoD sequence generation at frequencies higher than $1 \mathrm{~Hz}$, additional process parameter optimization and control would be needed. 


\section{Acknowledgments}

This work was supported by ARRS - the Slovenian Research Agency, and by the COST Action MP1106.

\section{References}

Albert, F., Pfeiffer, C., Schmidt, M., Geiger, M., Flössel, M., Michaelis, A., 2011. Laser Soldering and Laser Droplet Joining for Mechanical and Electrical Contacting of LTCC/PZT Laminates. Journal of Laser Micro/Nanoengineering 6, 75-80.

Conway, P., Fu, E., Williams, K., Williams, D., 2002. Precision high temperature lead-free solder interconnections by means of high-energy droplet deposition techniques. CIRP Annals - Manufacturing Technology 51, 177-180.

Cheng S. X., Li, T., Chandra, S., 2005. Producing molten metal droplets with a pneumatic drop-on-demand generator. Journal of Materials Processing Technology 159/3, 295-302.

Chun, J.-H., Passow, C.H., Suh, N.P., 1993. Droplet-Based Manufacturing. CIRP Annals - Manufacturing Technology 42, 235-238.

Dreizin, E.L., 1997. Droplet Welding: A New Technique for Welding Electrical Contacts. Welding Journal 4, $67-73$.

Govekar, E., Jerič, A., Weigl, M., Schmidt, M., 2009. Laser droplet generation: Application to droplet joining. CIRP Annals - Manufacturing Technology 58, 205-208.

Govekar, E., Klemenčič, J., Kokalj, T., Schmidt, M., Kastens, M., 2007. Progress in Laser Droplet Formation and Welding. Proceedings in Laser in der Elektronikproduktion \& Feinwerktechnik LEF 2007, Meisenbach Bamberg.

Horii, T., Kirihara, S., Miyamoto, Y., 2009. Freeform fabrication of superalloy objects by 3D micro welding. Materials and Design 30, 1093-1097.

Hu, J., Tsai, H.L., 2007. Heat and mass transfer in gas metal arc welding. Part I: The arc. International Journal of Heat and Mass Transfer 50, 833-846. 
Jahrsdörfer, B., Esser, G., Geiger, M., Govekar, E., 2003. Laser Droplet Weld - an innovative joining technology opens new application possibilities. Photon Processing in Microelectronics and Photonics II, Proceedings of SPIE, 4977, pp. 518-529.

Jerič, A., Grabec, I., Govekar, E., 2009. Laser droplet welding of zinc coated steel sheets. Science and Technology of Welding \& Joining 14, 662-668.

Jeromen, A., Kuznetsov, A., Govekar, E., 2014. Laser droplet generation from a metal foil. Physics Procedia 39, $585-593$

Kokalj, T., Klemenčič, J., Mužič, P., Grabec, I., Govekar, E., 2006. Analysis of the laser droplet formation process. Journal of Manufacturing Science and Engineering 128, 307-314.

Krese, B., Perc, M., Govekar, E., 2011. Experimental observation of a chaos-to-chaos transition in laser droplet generation. International Journal of Bifurcation and Chaos 21/6, 1689-1699.

Kuznetsov A., Jeromen, A., Govekar, E., 2014. Droplet detachment regimes in annular laser beam droplet generation from a metal wire. CIRP Annals - Manufacturing Technology, 63/1, 225-228.

Liu, H., 2000. Science and Engineering of Droplets: Fundamentals and Applications. New York: Noyes Publications/William Andrew Publishing.

Liu, Q., Orme, M., 2001. High precision solder droplet printing technology and the state-of-the-art. Journal of Materials Processing Technology 115, 271-283.

Liu, Q., Orme, M., 2001. On precision droplet-based net-form manufacturing technology. Proceedings of the Institution of Mechanical Engineers, Part B: Journal of Engineering Manufacture 215, 1333-1354.

Qi, L.-H., Chao, Y.-P., Luo, J., Zhou, J.-M., Hou, X.-H., Li, H.-J., 2012. Novel selection method of scanning step for fabricating metal components based on micro-droplet deposition manufacture. International Journal of Machine Tools and Manufacture 56, 50-58.

Quentin, U., Heberle, J., Held, C., Schmidt, M., 2013. Laser droplet brazing for electrical contacting of composite materials with integrated active elements. Procedia Materials Science 2, $181-188$. 
Shimasaki, S., Taniguchi, S., 2009. Formation of uniformly-sized droplets from capillary jet by electromagnetic force. Proceedings of Seventh International Conference on CFD in the Minerals and Process Industries, Melbourne, Australia.

Sohn, H., Yang, D.Y., 2005. Drop-on-demand deposition of superheated metal droplets for selective infiltration manufacturing. Materials Science and Engineering A 392, 415-421.

Terakubo, M., Oh, J., Kirihara, S., Miyamoto, Y., Matsuura, K., Kudoh, M., 2005. Freeform fabrication of titanium metal by 3D micro welding. Material science and Engineering A 402, 84-91.

Wehl, W., Wild, J., Lemmermeyer, B., 2003. A drop-on-demand metal jet printer for water bumping. Proceedings of 14th European Microelectronics and Packaging Conference \& Exhibition, Friedrichshafen, Germany.

Wu, C.S., Chen, M.A., Li, S.K., 2004. Analysis of excited droplet oscillation and detachment in active control of metal transfer. Computational Materials Science 31, 147-154.

Yamaguchi, K., Sakai, K., Yamanaka, T., Hirayama, T., 2000. Generation of three-dimensional micro structure using metal jet. Precision Engineering Journal 24, 2-8.

Zarzalejo, L.J., Schmaltz, K.S., Amon, C.H., 1999. Molten droplet solidification and substrate remelting in microcasting, Part I: numerical modeling and experimental verification. International Journal of Heat and Mass Transfer 34, 477-485.

Zhang, Y., Chen, Y., Li, P., Male, A.T., 2003. Weld deposition-based rapid prototyping: a preliminary study. Journal of Materials Processing Technology 135, 347-357.

Zhang, Y.M., Liguo, E., Walcott, B.L., 2002. Robust Control of Pulsed Gas Metal Arc Welding. Journal of Dynamic Systems, Measurement and Control, 124, 281-289.

\section{Figure captions}

Fig. 1. (a) Scheme of the annular LDG system; (b) Caustic of the annular laser beam; (c) Example of the annular laser beam intensity distribution. 
Fig. 2. Photo of the annular laser droplet generation head.

Fig. 3. Process phases and the related main process parameters: (a) Initial wire-end positioning; (b) Formation of a pendant droplet with minimum diameter; (c) Pendant droplet growth to the required diameter; (d) Pendant droplet neck positioning; (e) Pendant droplet detachment; (f) Laser pulse power $P(t)$ and wire feeding velocity $V_{\mathrm{w}}(t)$.

Fig. 4. An example of an IR camera record of the LDoDG process phases and stages : a) Formation of a pendant droplet with minimum diameter; b) Pendant droplet growth up to the required diameter; c) Pendant droplet neck positioning; d) Pendant droplet detachment; e) Droplet deposition.

Fig. 5. Examples of solidified pendant droplets with diameters ranging from 0.8 to $1.2 \mathrm{~mm}$.

Table 1. The relationships between $D_{\mathrm{rp}}$ and the corresponding $L_{\mathrm{we}}$, and $D_{\min }$ for $D_{\mathrm{w}}=0.6 \mathrm{~mm}$ and $h_{\mathrm{LB}}=0.8 \mathrm{~mm}$.

Fig. 6. Theoretical (solid line) and experimental (markers) correlations between the diameter $D_{\text {pdr }}$ of the pendant droplet and the fed wire length $L_{\mathrm{w}}$ to be melted.

Fig. 7. Relationship between the diameter of the pendant droplet $D_{\mathrm{pdr}}$ and the energy of the formation laser pulse $E_{\mathrm{fp}}$

Fig. 8. a) Surface temperature $T_{\mathrm{ws}}$ of the wire-end during the pendant droplet formation phase, b) the corresponding process parameters $P_{\mathrm{fp}}(t)$ (solid line) and $V_{\mathrm{w}}(t)$ (dashed line).

Fig. 9. Droplet detachment regimes and the initial vertical velocity $V_{\mathrm{v}, \mathrm{dr}}$ of the detached droplets vs. the distance $L_{\mathrm{dn}}$

Fig. 10. Dependence of the rest part diameter $D_{\mathrm{rp}}$ and the detached droplet diameter $D_{\mathrm{dr}}$ on the distance $L_{\mathrm{dn}}$.

Fig. 11. a) Temperatures $T_{\text {ws }}$ of the wire material at the laser beam focus spot during the droplet detachment phase, b) The detachment laser pulse $P_{\mathrm{dp}}$ power profiles.

Fig. 12. Droplet temperature $T_{\mathrm{dr}}$ vs. droplet diameter $D_{\mathrm{dr}}$.

Fig. 13. Lateral droplet centre position of detached droplets at a stand-off distance $d_{\mathrm{s}}=1.5 \mathrm{~mm}$ versus the distance $L_{\mathrm{dn}}$. 
Fig. 14. Characteristic times of the LDoDG process duration vs. droplet diameter $D_{\mathrm{dr}}$.

Fig. 15. Examples of deposited droplets with increasing diameter $D_{\mathrm{dr}}$, ranging from 0.85 to $1.25 \mathrm{~mm}$ and selected enlarged droplets.

Fig. 16. Examples of top and side views of in line deposited droplets with diameters of $D_{\mathrm{dr}}=0.85,1.05$, and $1.25 \mathrm{~mm}$.

Fig. 17. Examples of various $2 \mathrm{D}$ patterns created by the deposition of droplets with different diameters $D_{\mathrm{dr}}$. 